\title{
The Orbit Space of a Kleinian Group: Riley's Modest Example
}

\author{
By Matthew A. Grayson
}

In the previous paper, Robert Riley [4] and his computer file Poincare found a fundamental domain for the action of a discrete group $G$ of isometries of hyperbolic space $\mathbb{H}^{3}$ generated by three parabolics. In this paper, we show that the orbit space $\mathbb{H}^{3} / G$ is homeomorphic to a complement $S^{3}-k^{*}$, where $k^{*}$ is $k$ union a point and where $k$ is the $(3,3,3)$ pretzel knot. Furthermore, $\mathbb{H}^{3} / G$ is equipped with an infinite volume hyperbolic orbifold structure. This should not be confused with the complete finite volume hyperbolic structure on $S^{3}-k$. A neighborhood of $k$ in $\mathbb{H}^{3} / G$ is not the quotient of a horoball by a group of parabolic isometries with common fixed point. It is instead the quotient of a neighbourhood of the domain of discontinuity for $G$. In addition, $G$ has elliptic elements of order three which give rise to three singular axes in the hyperbolic structure. These three axes meet at a point at infinity in $\mathbb{H}^{3} / G$. This accounts for the additional missing point in $S^{3}-k^{*}$. A neighbourhood of this point is the quotient of a horoball by the Euclidean $(3,3,3)$ triangle group. For a treatment of hyperbolic and orbifold structures, see Thurston [5].

The computer output form Riley's program consists of a picture of the fundamental domain $\mathscr{D}$, and a data output giving the face pairings and face pairing transformations. For this paper, only the picture of $D$ appearing in Section 3 of the preceding paper is used, as the information that it contains is sufficient to determine the face pairings uniquely. As a result, our labellings are different from those appearing in the data output, but the face pairings are the same. To show the homeomorphism $\mathbb{H}^{3} / G=S^{3}-k^{*}$, we will glue up by identifying paired faces. The geometric structure will then arise as a direct consequence of the gluing.

The domain $\mathscr{D}$ is an infinite volume hyperbolic polyhedron. In the upper half space model $\mathcal{Q}^{3}$ it lies between two $E H$-planes parallel to the imaginary axis of the boundary complex plane $\pi_{0}$. The E-closure of $\mathscr{Q}, \overline{\mathcal{D}}$, contains three subsets of $\pi_{0}$. Two of these are compact, and are labelled $Y$ and $Z$. The third region, labelled $X$, has connected closure in $\pi_{0}^{*}$ and intersects any neighborhood of the point $\{\infty\}$.

Figure 1 shows a slightly altered version of the original computer drawing with added labellings of faces and edges. Some of the edges of $\mathscr{D}$ are EH-lines and, as such, they do not appear on the computer drawing. This poses no difficulty, as these edges will be subsumed by the first gluing step and will play no further part in the discussion.

Received April 29, 1982.

1980 Mathematics Subject Classification. Primary 57N10, 30F40, 57M25. 


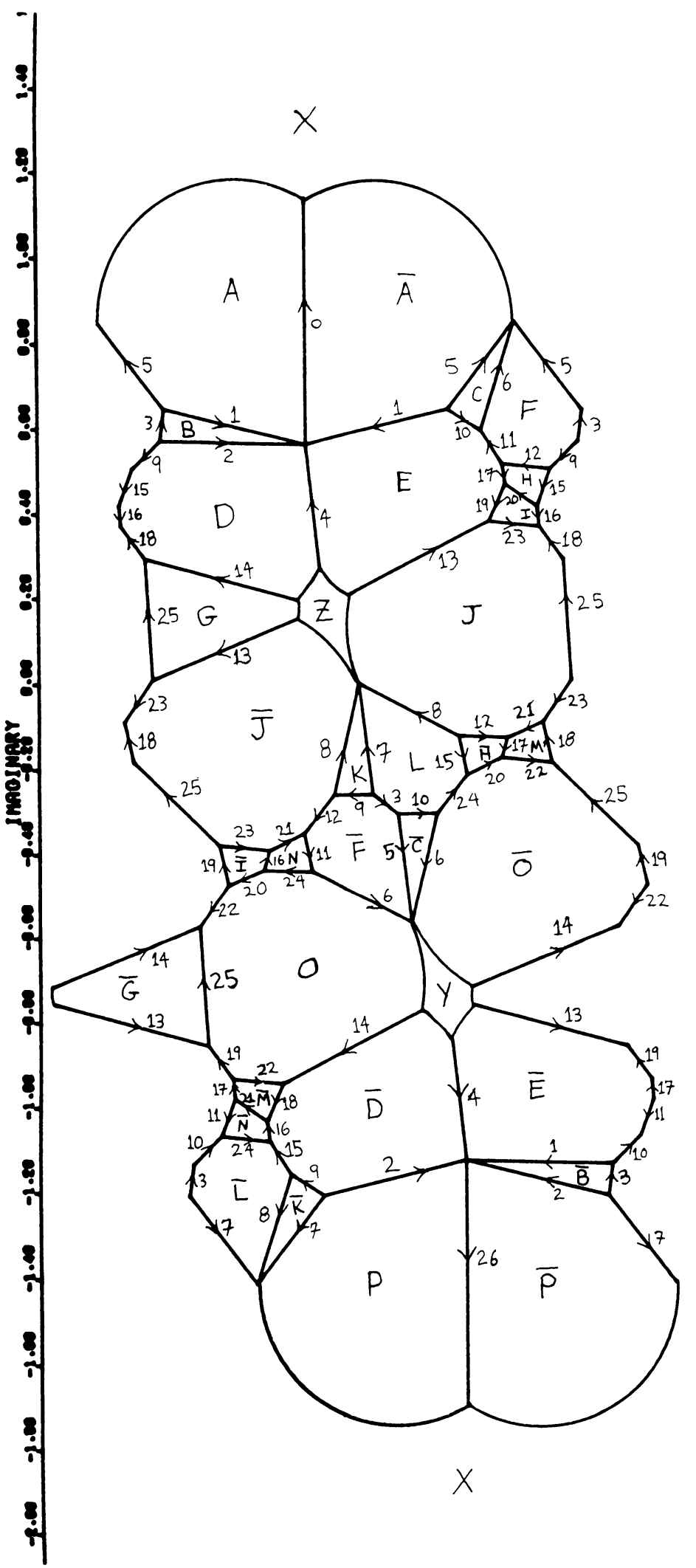

FIGURE 1. The fundamental domain $\mathscr{D}$ 


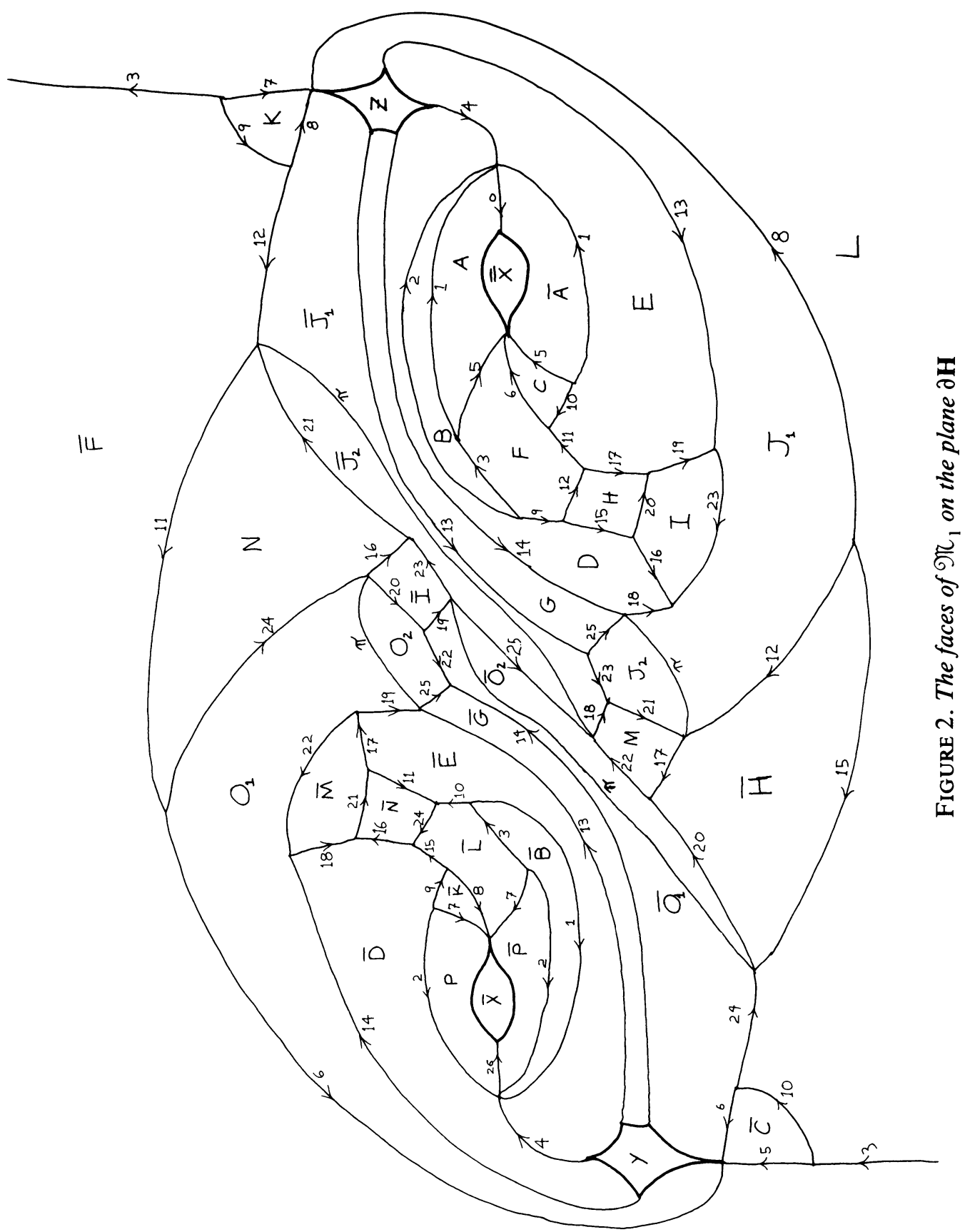


The first gluing step is induced by the parabolic isometry $\mathbf{A}=\left(\begin{array}{ll}1 & 1 \\ 0 & 1\end{array}\right)$ acting on $\mathcal{Q}^{3}$. $\mathcal{Q}^{3} /(\mathbf{A})$ is then an open solid torus. $\Re_{1}=\mathscr{D} /(\mathbf{A})$ is obtained by identifying the leftand right-hand sides of $\mathscr{D}^{.} \mathfrak{T}_{1}$ is a closed solid torus minus a closed subset of its boundary, namely $X \cup Y \cup Z \cup\{\infty\}$. Even though the regions $X, Y$ and $Z$ are not part of $\Re_{1}$, these labels will be retained to indicate the missing pieces of the boundary of the closed solid torus. Viewed as such, the region $X$ in $\Re_{1}$ is now a cylinder. For a more detailed treatment of the compactification of fundamental polyhedra, the reader is referred to Riley [2], [3].

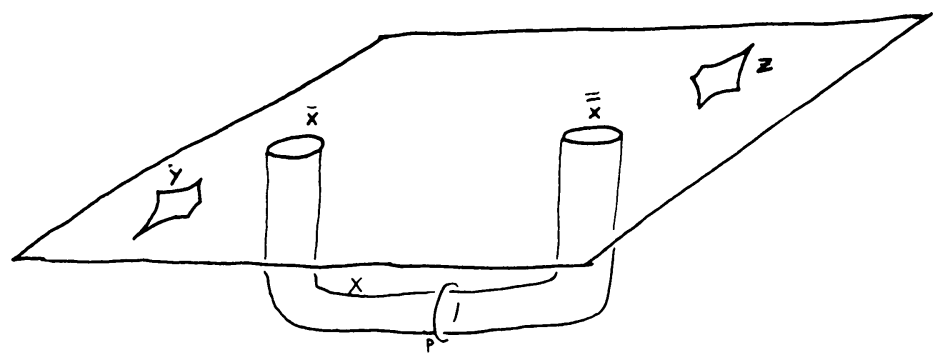

FIGURE 3. $\Re_{1}$ and the tube running through $\mathbf{H}$

For the rest of the discussion, it is convenient to represent $\mathfrak{R}_{1}$ as follows. A solid torus is homeomorphic to a ball minus a 1-handle, which can be drawn as a closed half space $\mathbf{H}$ minus a 1-handle. The advantage of this description is that it keeps the faces of $\mathfrak{R}_{1}$ on a plane $\partial \mathbf{H}$, and the cylinder $X$ forms the boundary of the removed 1-handle.

Figure 2 is a picture of the plane bounding the half space $\mathbf{H}$. The cylinder $X$ has two boundary components on this plane; they are labelled $\bar{X}$ and $\overline{\bar{X}}$. Edge 3 passes out of the picture, through the point $\{\infty\}$, and back in. Note that this is the edge passing through the center of Figure 1. A diagram showing the positions of $X, \bar{X}, \bar{X}, Y$ and $Z$ is given in Figure 3. In the hyperbolic structure induced on $\Re_{1}$ from $\Phi /(\mathbf{A})$, the tube $X$ is pinched in the middle. This is because $\mathbf{A}$ fixes the point at infinity. In the figures, this occurrence will be denoted by a circle labelled $p$ for parabolic.

From here, the argument consists of identifying paired faces in a suggested order. The map which identifies, say, faces $C$ and $\bar{C}$ will be written $(C, \bar{C})$. At each stage of the identification, paired faces will sink into the interior below the plane $\partial \mathbf{H}$, leaving only unidentified faces on the surface.

Figure 2 suggests that the gluing may begin with the pairs $A, \bar{A}$ and $P, \bar{P}$. Identifying these pairs has an interesting consequence. By Riley's angle sum trick [4], we know that the dihedral angles of $\mathscr{D}$ summed over the occurrences of any edge yield a rational multiple of $2 \pi$. For the edges labelled 0,4 and 26 , this sum is $2 \pi / 3$; for all other edges it is $2 \pi$. Identifying $A, \bar{A}$ makes a neighborhood of edge 0 isometric with a neighborhood of the EH-line between 0 and $\{\infty\}$ in $\mathcal{U}^{3} /(z \rightarrow \omega z)$, $\omega$ a primitive cube root of unity. Such a structure is an example of an orbifold structure as defined in Thurston [5]. In the figures, orbifold axes are denoted by solid arcs. 

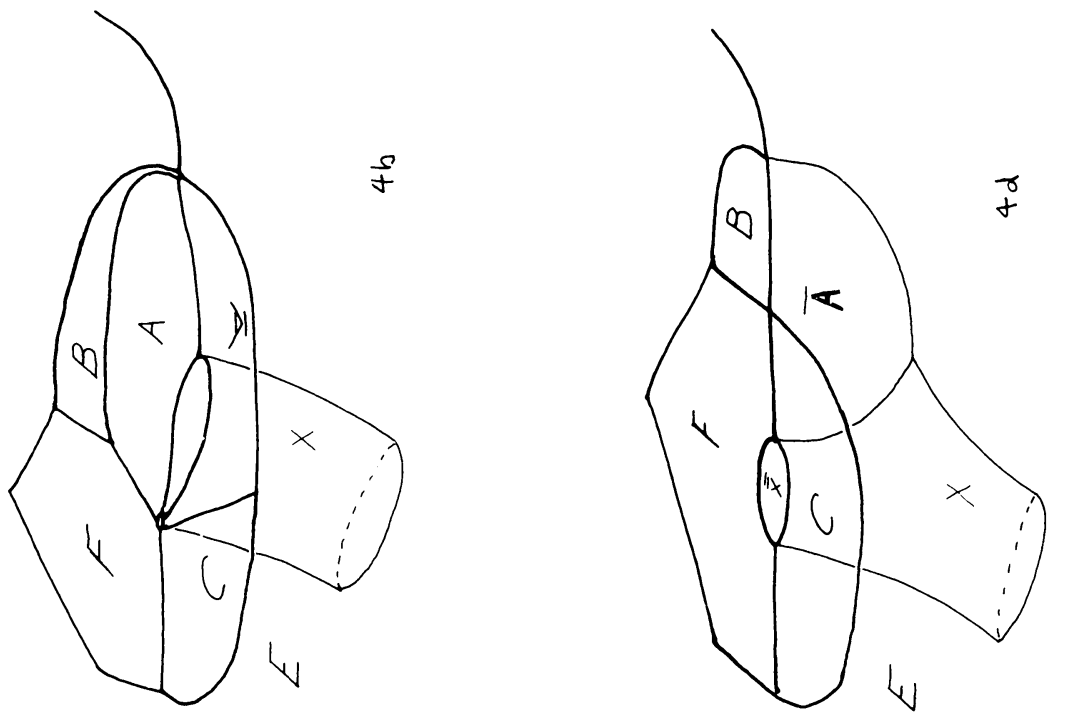

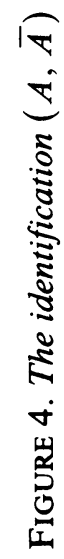
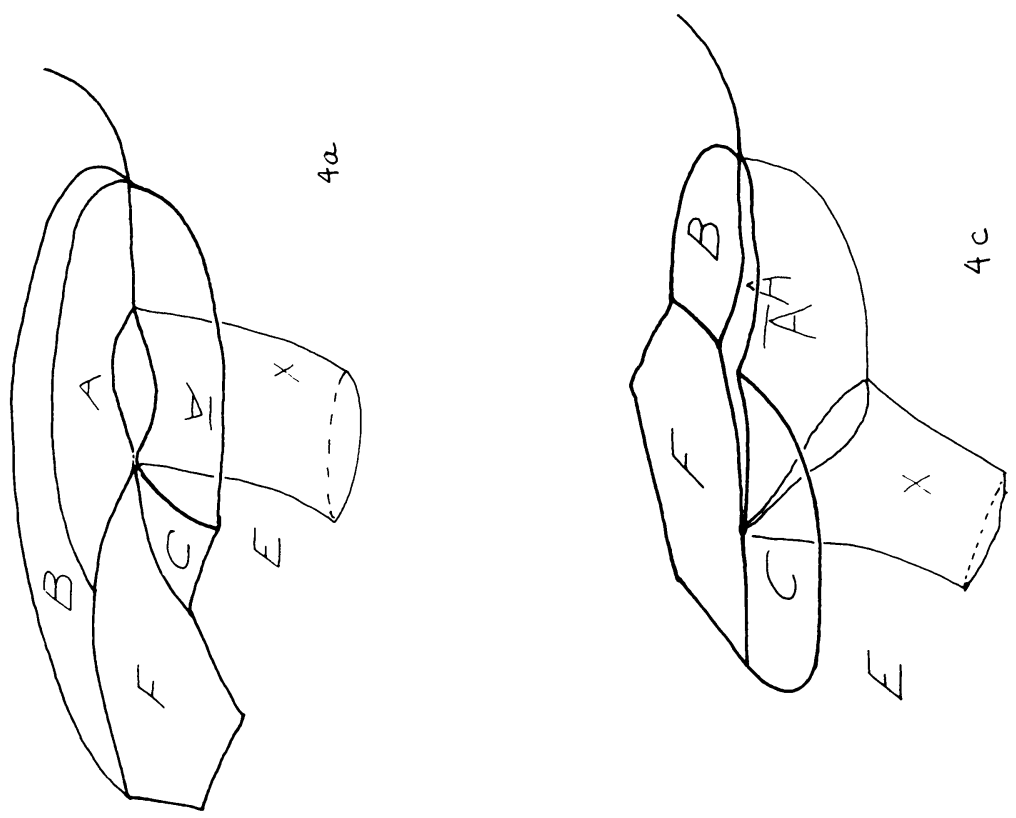


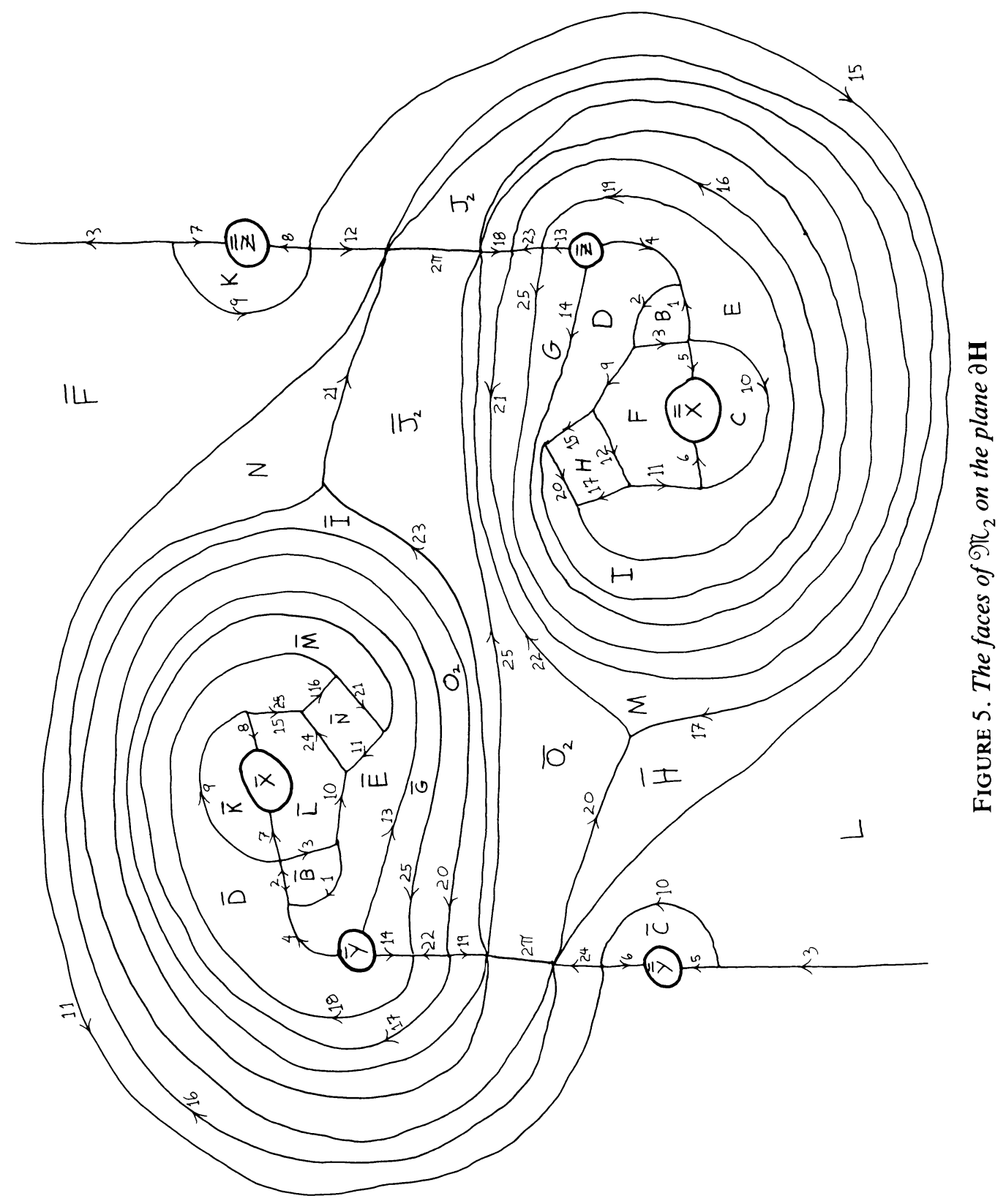


The map $(A, \bar{A})$, which can be visualized as closing the pages of a book with, say, $A$ on the right-hand page and $\bar{A}$ on the left, actually identifies the circle $\overline{\bar{X}}$ with itself, leaving only a point touching the plane $\partial \mathbf{H}$. For the sake of the figures, this point will be left a circle, and it will be marked later with a parabolic $p$. A detailed description of the identification $(A, \bar{A})$ is given in Figure 4. A similar discussion holds for $(P, \bar{P})$.

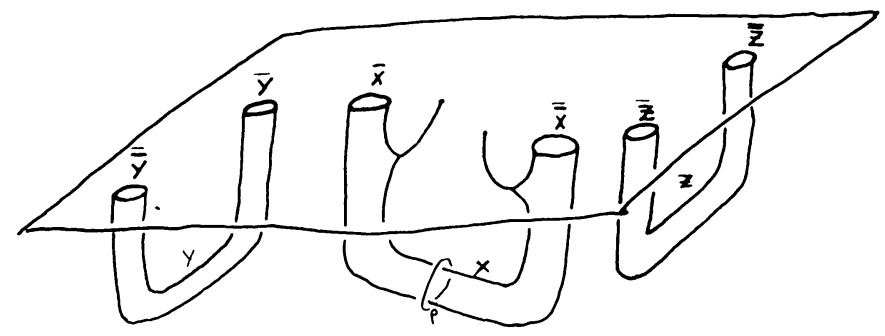

Figure 6. $\Re_{2}$, its tubes and orbifold axes in $\mathbf{H}$

At this point, a more complicated gluing is possible. Faces $O, \bar{O}$ and $J, \bar{J}$ could be identified in a manner similar to $A, \bar{A}$ and $P, \bar{P}$. Unfortunately, each of these faces shares an edge in common with its counterpart, edges 22 for $O, \bar{O}$ and 23 for $J, \bar{J}$. To overcome the conceptual difficulty involved in such a gluing, we identify only part of each side. In Figure 2, each of the four faces $O, \bar{O}, J$ and $\bar{J}$ have been divided in two by an edge marked $\pi$ to indicate the dihedral angle concentrated at this edge. It is now a simple matter to identify the faces $O_{1}, \bar{O}_{1}$ and $J_{1}, \bar{J}_{1}$ by the maps $(O, \bar{O})$ and $(J, \bar{J})$. This identification leaves the faces $\mathrm{O}_{2}, \bar{O}_{2}$ and $J_{2}, \bar{J}_{2}$ separated by an edge containing a full $2 \pi$ of dihedral angle. Geometrically, the faces $\mathrm{O}_{2}, \overline{\mathrm{O}}_{2}$, say, are adjacent and tangent, but not yet identified. Notice, also, that the maps $(O, \bar{O})$ and $(J, \bar{J})$ fold the regions $Y$ and $Z$ into tubes running through the half space $\mathbf{H}$. As before, the ends of these tubes will be labelled $\bar{Y}, \overline{\bar{Y}}$ and $\bar{Z}, \overline{\bar{Z}}$. The resulting quotient space, $\mathfrak{T}_{2}=\mathfrak{K}_{1} /(A, \bar{A}),(P, \bar{P}),\left(O_{1}, \bar{O}_{1}\right),\left(J_{1}, \bar{J}_{1}\right)$ is shown in Figures 5 and 6.

To glue any further, it is necessary to move around the circles $\bar{X}, \overline{\bar{X}}, \bar{Y}, \overline{\bar{Y}}, \bar{Z}, \overline{\bar{Z}}$. This has the effect of simplifying the diagram, as well as tangling the tubes running through $\mathbf{H}$. In this way, we begin to obtain our manifold as the complement of some knot or link in $S^{3}$. Figures 7 and 8 show the end result of this manipulation. No identifications have been performed.

Now we can easily identify $\mathrm{O}_{2}, \overline{\mathrm{O}}_{2}$ and $\mathrm{J}_{2}, \bar{J}_{2}$. Previous identifications of edges 22 and 23 allow $M, \bar{M}$ and $I, \bar{I}$ to be identified at the same time. Circles $\overline{\bar{Y}}$ and $\bar{Z}$ move around the picture as a result. $\Re_{3}=\Re_{2} /\left(O_{2}, \bar{O}_{2}\right),\left(J_{2}, \bar{J}_{2}\right),(M, \bar{M}),(I, \bar{I})$ is shown in Figures 9 and 10. 


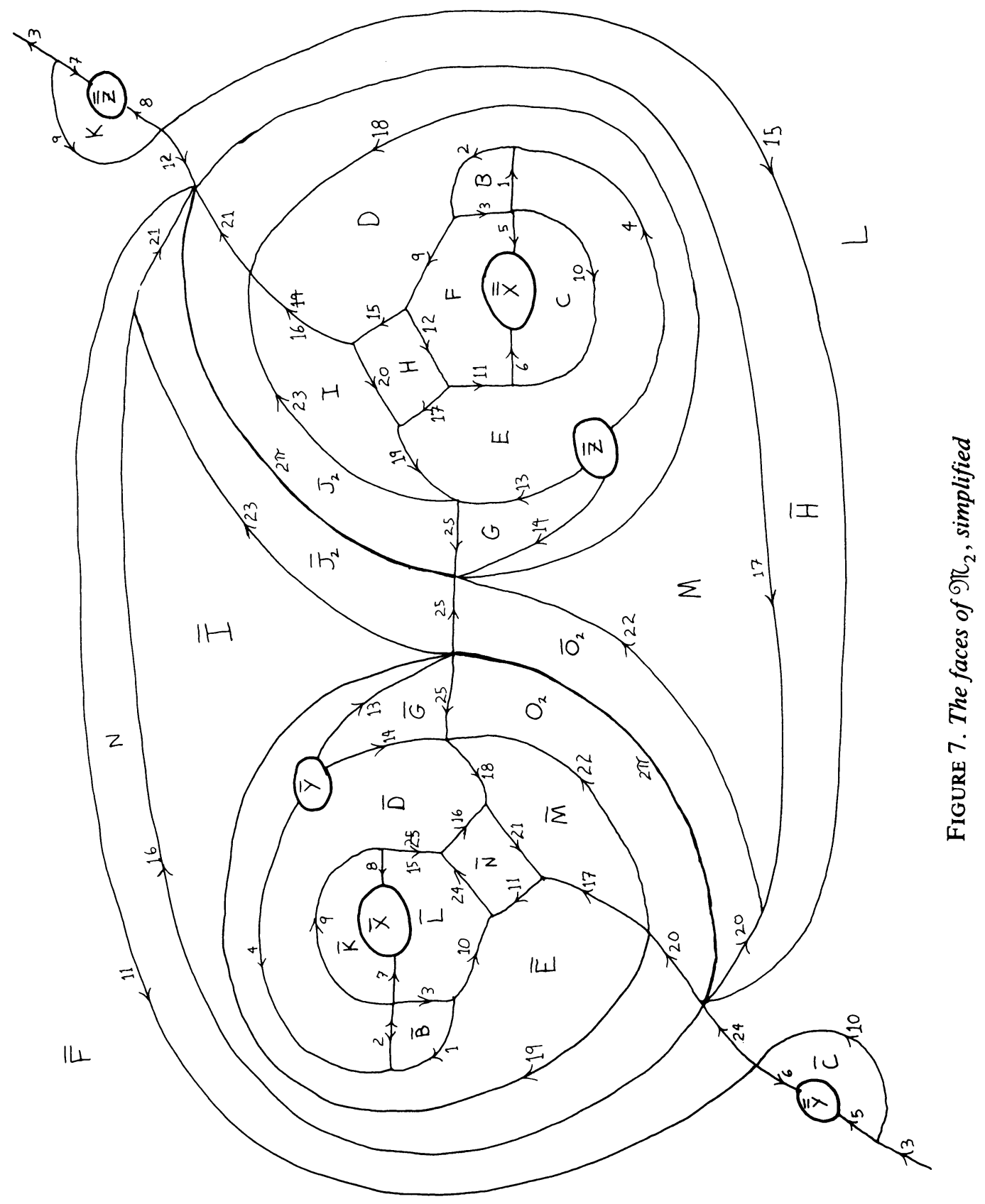


Looking at Figure 9, we realize that all occurrences of edges 18, 19 and 25 have been identified, that is, there is a full $2 \pi$ of dihedral angle concentrated at each of these edges. We can then identify faces $G, \bar{G}, E, \bar{E}, D, \bar{D}$ and $B, \bar{B}$. This joins tubes $Y$ and $Z$ at the circles $\bar{Y}$ and $\bar{Z}$ and forms a new orbifold axis, edge 4 . This is seen in Figures 11 and 12 . In a like manner, we can identify $N, \bar{N}$ and $H, \bar{H}$. Figures 13 and 14 show what is left, and without further manipulation, we identify $F, \bar{F}, L, \bar{L}, K, \bar{K}$ and $C, \bar{C}$, identifying tubes $X$ and $Z$ at $\bar{X}$ and $\overline{\bar{Z}}$ and tubes $X$ and $Y$ at $\overline{\bar{X}}$ and $\overline{\bar{Y}}$, completing the gluing. Figures 15 and 16 show two views of the resulting knot complement, its parabolic circles and orbifold axes. The three orbifold axes meet at a point which, from angle considerations, must be at infinity. We could have made this observation earlier, but as it is not obvious from the original figures that both endpoints of edge 4 lie on the plane $\pi_{0}$, we decided to withhold this assertion until it could be seen more clearly. A neighborhood of this point in $\mathbb{H}^{3} / G$ is homeomorphic to the product of an open interval with a three cornered sphere, in this case $S^{2}$ with a Euclidean structure everywhere except at three points, each of which has a cone angle of $2 \pi / 3$. The other boundary component, the torus, has a hyperbolic structure induced by the conformal structure on the domain of discontinuity for a Kleinian group. The conformal structure is that of a Riemann surface with three nodes alternating with three elliptic points of order three.

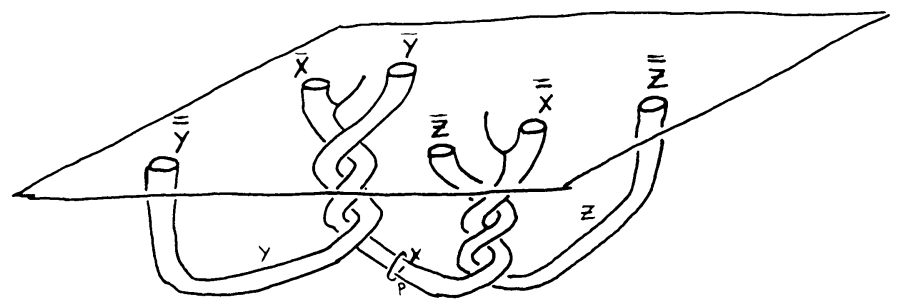

FIGURE $8 . \Re_{2}$ after simplification

We have really constructed this knot by its three bridge presentation. In general, the technique of starting with $n$ tubes running through half space and shuffling their $2 n$ boundary components will yield the complement of an $n$ bridge link. For the pretzel knot $(3,3,3)$, Riley has produced a sequence of groups $G_{2 n}, n \geqslant 2$, and fundamental domains $\mathscr{D}_{2 n}$. The $G$ in this paper is $G_{6}$ of this sequence. Each case can be treated in exactly the same way, the only differences being the orders of the orbifold axes. For $G_{2 n}$, the axes have order $n$, and the resulting quotient has a boundary component homeomorphic to $S^{2}$ with the structure of a hyperbolic 2-orbifold with three points, each an elliptic point of order $n$. This holds for all $n$ greater than 3. For $n$ equals 2 , there is only one boundary component, for the three axes meet at a finite point. Unfortunately, no general proof of the discreteness of these kinds of groups has been found, and presently, ad hoc methods are necessary. For further examples, the reader is referred to Riley [1]. 


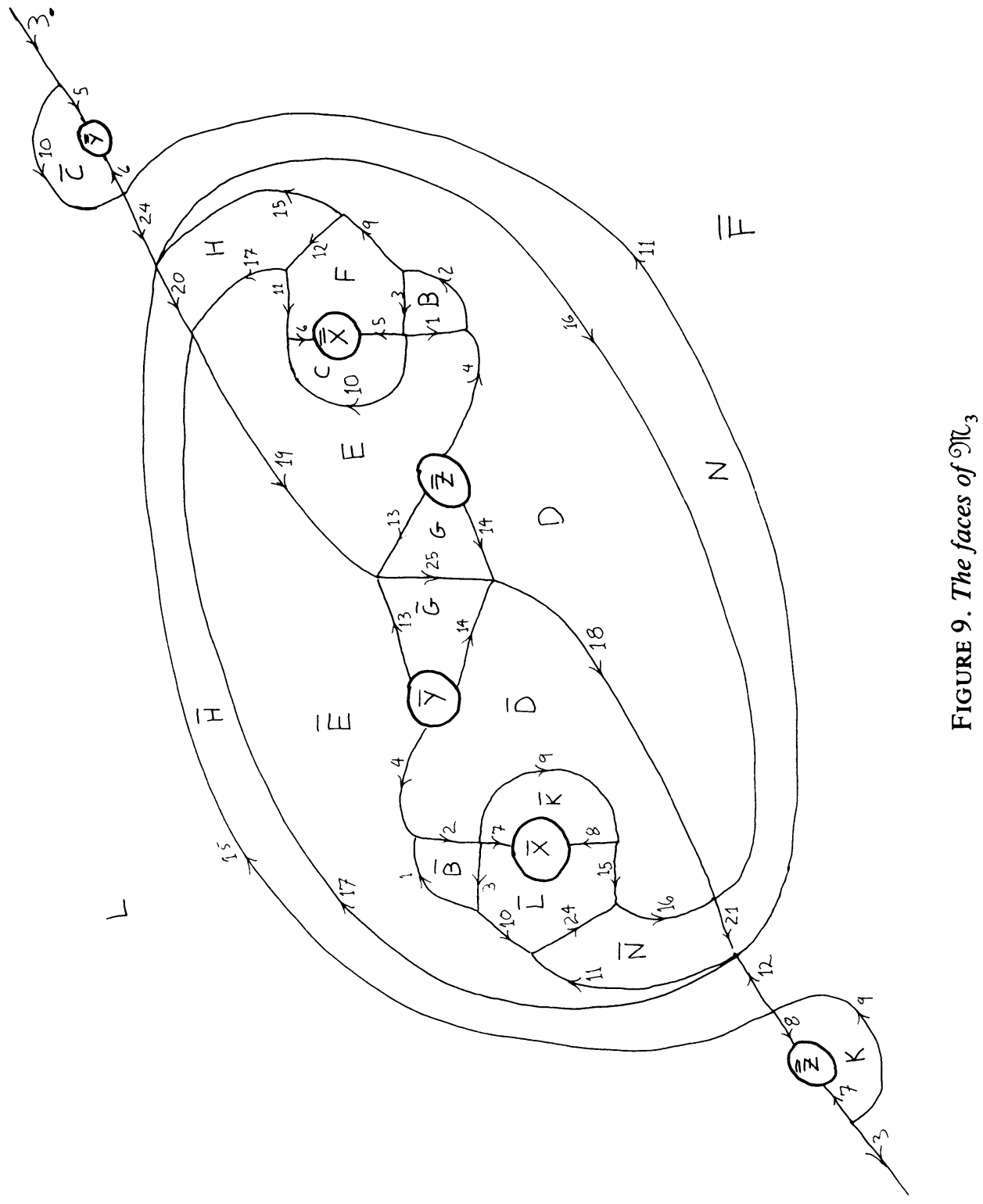




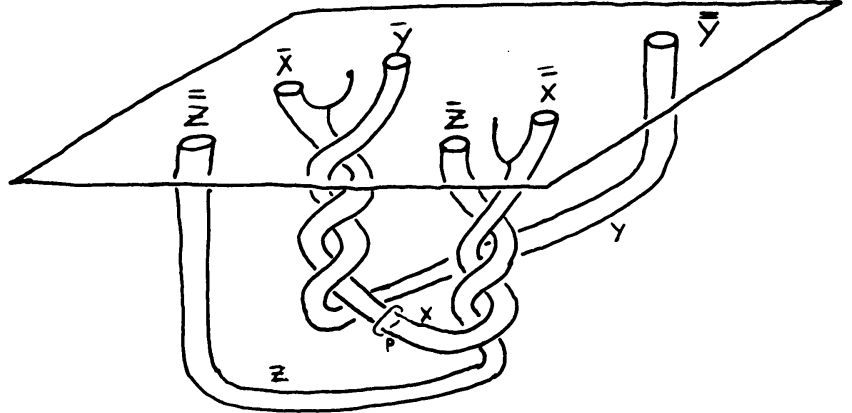

FIGURE 10. $\Re_{3}$, its tubes and orbifold axes in $\mathbf{H}$

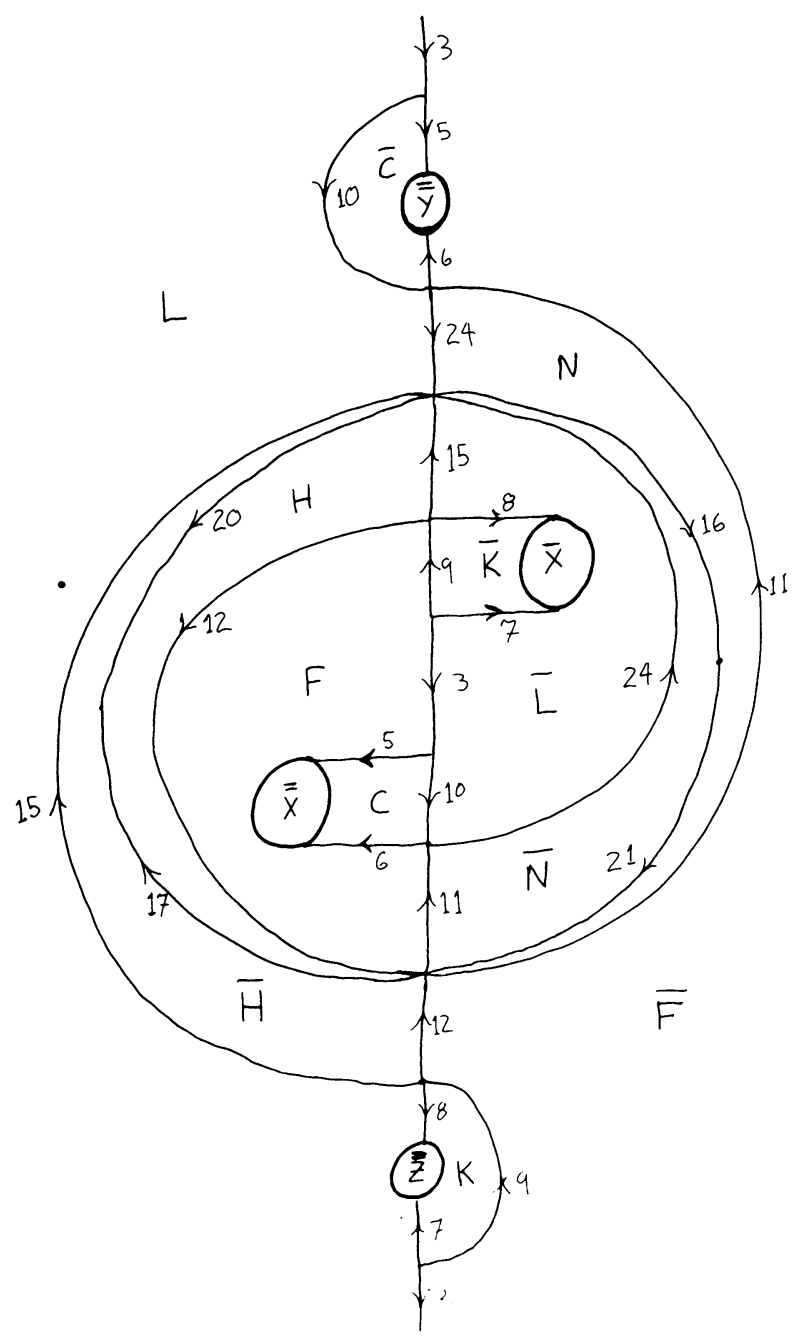

FIGURE 11. The faces of $\mathfrak{M}_{4}=\mathfrak{M}_{3} /(G, \bar{G}),(E, \bar{E}),(D, \bar{D}),(B, \bar{B})$ 


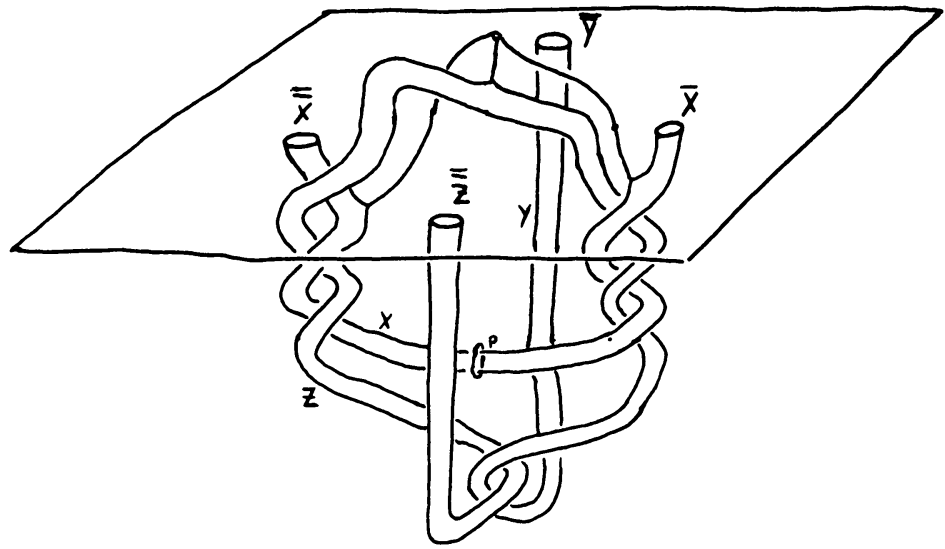

FIGURE 12. $\Re_{4}$ in $\mathbf{H}$

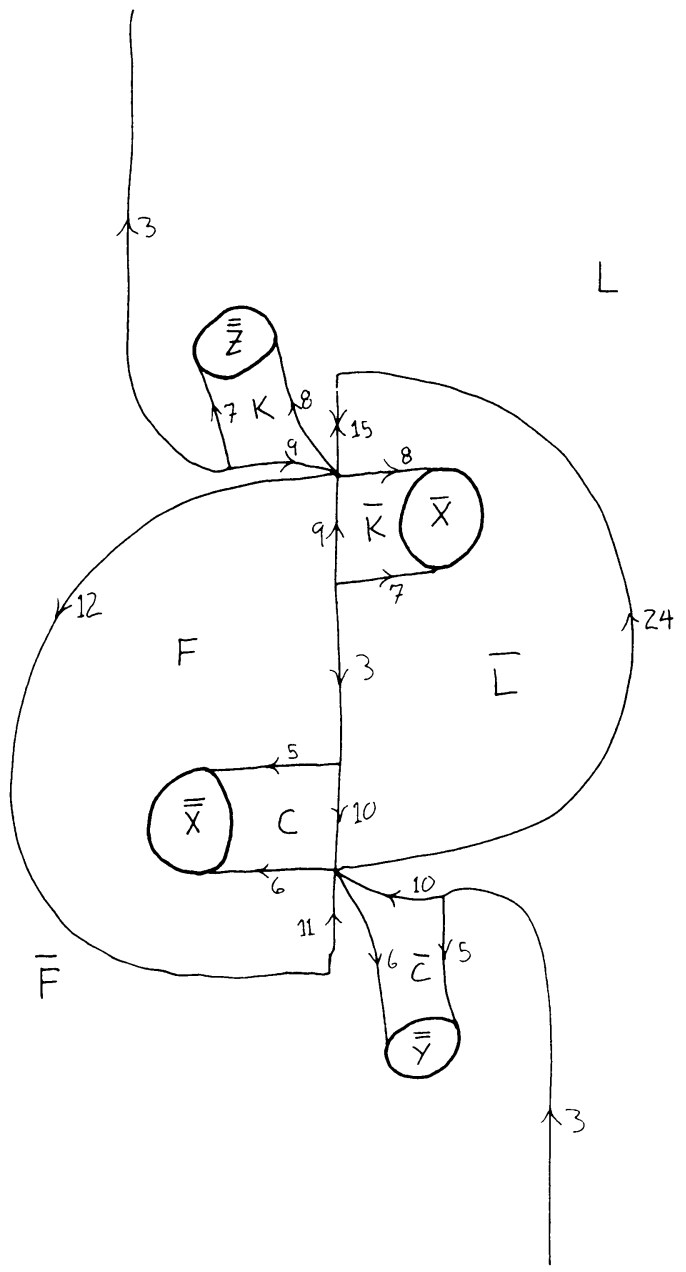

FIGURE 13. $\Re_{5}=\Re_{4} /(N, \bar{N}),(H, \bar{H})$ 


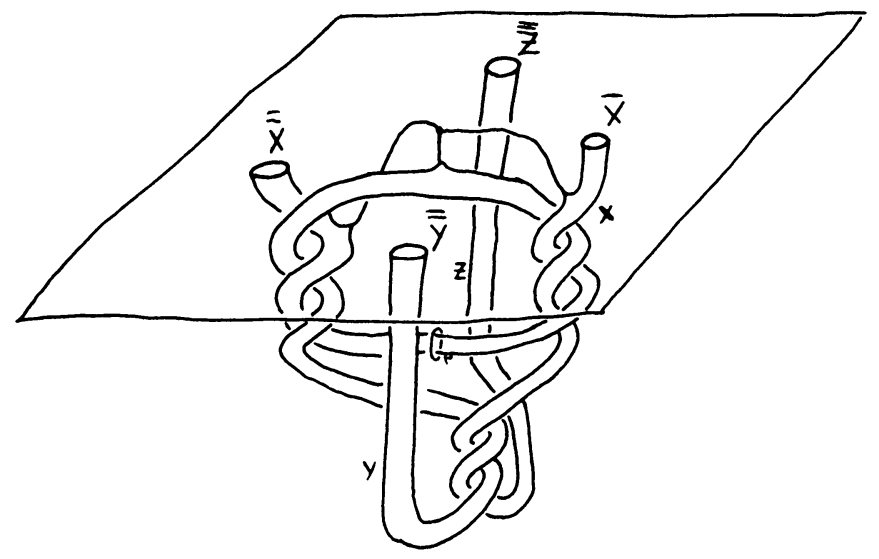

FIGURE 14. $\Re_{5}$

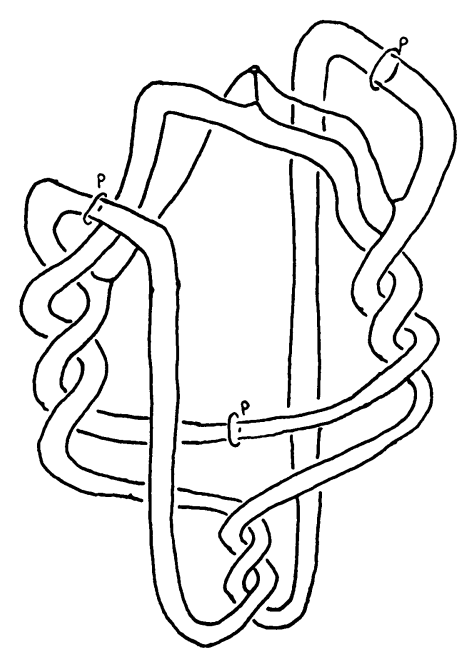

FIGURE $15 . \mathbb{H}^{3} / G$

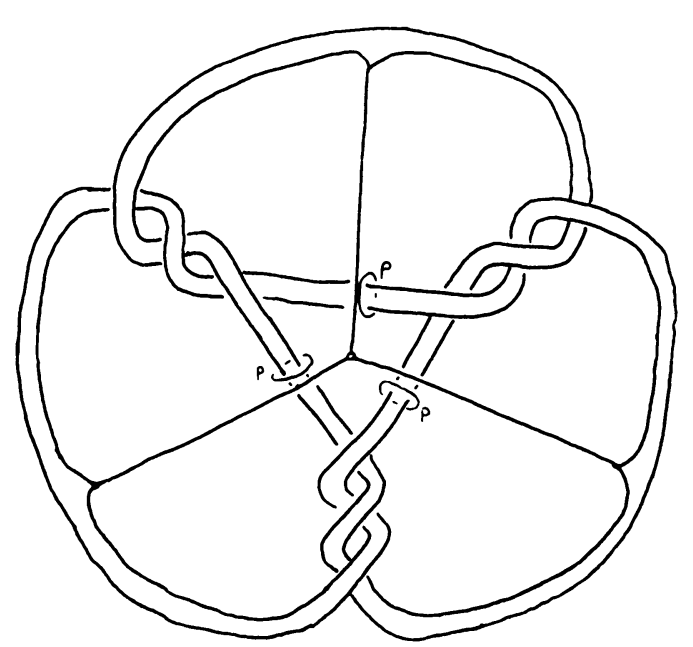

FIGURE 16. $\mathrm{H}^{3} / G$, another view 
Mathematics Department

Princeton University

Princeton, New Jersey 08544

Mathematics Department

University of Colorado

Boulder, Colorado 80302

1. R. Riley, Projective Representations of Link Groups, Ph. D. Thesis, University of Southampton, England, 1979.

2. R. Riley, “Discrete parabolic representations of link groups," Mathematika, v. 22, 1975, pp. $141-150$.

3. R. RILEY, "An elliptical path from parabolic representations to hyperbolic structures," in Topology of Low' Dimensional Manifolds (R. Fenn, ed.), Lecture Notes in Math., vol. 722, Springer-Verlag, Berlin and New York, 1979.

4. R. Riley, "Applications of a computer implementation of Poincarés Theorem on fundamental polyhedra," Math. Comp., v. 40, 1983, pp. 607-632.

5. W. Thurston, Three Dimensional Geometry and Topology, Princeton Univ. Press, 1983. (To appear) 